\title{
Evaluation of Xpert MTB/RIF Ultra for Diagnosis of Extrapulmonary Tuberculosis: A Retrospective Analysis in a Low-Tuberculosis Prevalence Setting
}

\author{
Annalisa Del Giudice ${ }^{1}$, Rossella Perna ${ }^{1}$, Teresa Baldoni1, Francesco Scarano ${ }^{2}$, \\ Annunziata De Rosa' ${ }^{2}$, Agostina Pontarelli' ${ }^{2}$, Mario De Marco², Martha Alicia Falca1, \\ Clelia Di Tella1, Luigi Atripaldi', Sergio Alfonso Harari ${ }^{3}$, Andrea Bianco ${ }^{4}$, Roberto Parrella2* \\ ${ }^{1}$ UOC Microbiologia e Virologia, Hospital Cotugno, AORN Ospedali dei Colli, Naples, Italy \\ ${ }^{2}$ UOC Respiratory Infectious Diseases Unit, Hospital Cotugno, AORN Ospedali dei Colli, Naples, Italy \\ ${ }^{3}$ Department of Medical Sciences San Giuseppe Hospital MultiMedica IRCCS and Department of Clinical Sciences and \\ Community Health, University of Milan, Milan, Italy \\ ${ }^{4}$ Department of Translational Medical Sciences, University of Campania “L. Vanvitelli”/Hospital Monaldi, Naples, Italy \\ Email: ^annalisa.delgiudice@ospedalideicolli.it, *roberto.parrella@ospedalideicolli.it
}

How to cite this paper: Del Giudice, A., Perna, R., Baldoni, T., Scarano, F., De Rosa, A., Pontarelli, A., De Marco, M., Falca, M.A., Tella, C.D., Atripaldi, L., Harari, S.A., Bianco, A. and Parrella, R. (2021) Evaluation of Xpert MTB/RIF Ultra for Diagnosis of Extrapulmonary Tuberculosis: A Retrospective Analysis in a Low-Tuberculosis Prevalence Setting. Journal of Tuberculosis Research, 9 , 219-229.

https://doi.org/10.4236/jtr.2021.93021

Received: April 26, 2021

Accepted: September 24, 2021

Published: September 27, 2021

Copyright $\odot 2021$ by author(s) and Scientific Research Publishing Inc. This work is licensed under the Creative Commons Attribution International License (CC BY 4.0).

http://creativecommons.org/licenses/by/4.0/ (c) (i) Open Access

\begin{abstract}
Background: Extrapulmonary tuberculosis (EPTB) remains difficult to diagnose because the clinical specimens to be examined are often paucibacillary and obtained with difficulty from inaccessible sites. An updated Xpert ${ }^{\circledR}$ MTB/RIF Ultra (Ultra) test has been designed and licensed to improve sensitivity in the detection of Mycobacterium tuberculosis complex. The aim of the present study is to evaluate the performance of Ultra assay for the clinical diagnosis of EPTB in a low tuberculosis prevalence country. Methods: A retrospective analysis was performed at "A. O dei Colli" of Naples on consecutive extrapulmonary specimens for EPTB across a three-year period. All different types of extrapulmonary specimens were tested for EPTB by smear microscopy, culture and Ultra assay in accordance with relevant guidelines. Results: A total of 606 EPTB samples, 561 culture negative EPTB and 45 culture positive EPTB were included. Using culture as reference standard, the overall sensitivities and specificities of Ultra assay were 95.6\% (95\% CI 84.8 99.5) and $97.5 \%$ (95\% CI 95.8 - 98.6) respectively. Sensitivity and specificity of Ultra for individual category of specimens were also performed. Conclusion: In a low-tuberculosis prevalence setting, Ultra assay confirms to have a good performance in the diagnosis of EPTB for all different extrapulmonary samples.
\end{abstract}




\section{Keywords}

Extrapulmonary Tuberculosis, Molecular Diagnosis, Mycobacterium tuberculosis, Xpert $^{\circledR}$ MTB/RIF Ultra

\section{Introduction}

Tuberculosis (TB) remains a critical clinical and public health issue worldwide. The latest data provided by the World Health Organisation (WHO) shows that in 2019 about 10 million people developed Tuberculosis with 1.4 million deaths. At the national level, the annual incidence rate of Tuberculosis ranged from less than 5 to more than 500 new and relapsed cases per 100,000 inhabitants [1]. The countries that had a low incidence of tuberculosis ( $<10$ cases per 100,000 population per year) in 2019 were 54 located mostly in the WHO Region of the Americas and the European Region plus a few countries in the Eastern Mediterranean and Western Pacific regions [1]. In Italy, the recorded notification rate in 2017 was 6.5 cases per 100,000 people [2] indeed considered a low TB country. In the foreign-born population since 2009 , more than $50 \%$ of the Tuberculosis cases notified each year and most of the cases of multidrug-resistant Tuberculosis (MDRTB) have been registered [2]. TB mostly affects the lungs (pulmonary TB, PTB) but can also affect many other different sites such tissues, lymph nodes and blood (extrapulmonary TB, EPTB). EPTB diagnosis remains a great challenge due to the diversity of symptoms with which EPTB may present, the low level of suspicion of clinicians and the difficulty in obtaining adequate samples for $\mathrm{M}$. $\mathrm{Tu}$ berculosis complex (MTC) detection. The delayed diagnosis of EPTB may lead to inappropriate treatment [3] [4], indeed, patients are often started on empirical antituberculosis treatment based on composite reference standard, including clinical signs and symptoms and radiological findings [5].

In 2010 the revolutionary Polymerase chain reaction (PCR)-based test, $\mathrm{Xpert}^{\mathbb{R}}$ MTB/RIF (Xpert; Cepheid, Sunnyvale, CA) was introduced. It consists of a rapid, semi-quantitative, in vitro nested real-time PCR assay for the simultaneous detection of MTC DNA and rifampicin resistance-associated $r p o B$ gene mutations within two hours [6]. Previous studies have shown that Xpert has a good performance in the diagnosis of PTB with smear positive sputum specimens [7]. In contrast, Xpert had a high specificity, but limited sensitivity in smear negative PTB samples and for the diagnosis of EPTB [7] [8]. To improve the performance of Xpert, an upgraded version of the assay, Xpert ${ }^{\circledR}$ MTB/RIF Ultra, has been developed by Cepheid (Ultra; Sunnyvale, USA) [5] [6]. The Ultra cartridge showed an increased sensitivity compared to the Xpert cartridge for the detection of MTC in specimens with low numbers of bacilli, especially in smear-negative, in HIV/ TB co-infected, in paediatric specimens and in extra-pulmonary specimens [9]. The incorporation of two new targets IS1081 and IS6110 significantly increases the sensitivity of the Ultra assay, enabling to detect 16 colony-forming units (CFU/ 
$\mathrm{mL}$ ), in contrast to the $114 \mathrm{CFU} / \mathrm{mL}$, limit of detection of Xpert ${ }^{\circledR}$ MTB/RIF. To achieve this category result, named "trace", at least one of the probes must be positive for IS1081 or IS6110 with cycle thresholds below 37 cycles and have no more than one rpoB probe with cycle thresholds below 40 cycles [10]. Since March 2017 the WHO has recommended Ultra as a replacement for Xpert in all circumstances [11].

So far, most of the studies have compared the performance of Ultra to Xpert on pulmonary samples [12] [13] [14] or have investigated Ultra for diagnosing EPTB in TB endemic countries [5] [15] [16]. The aim of our study is to provide an evaluation of Xpert Ultra in a routine diagnostic mycobacteriology laboratory of a large metropolitan city of South Italy, Naples that is considered a low tuberculosis setting ( $<10$ cases per 100,000 population per year) [1].

\section{Material and Methods}

\subsection{Study Design and Sample Inclusion}

This study was conducted at the Laboratory of Microbiology and Virology of "A.O dei Colli" (Naples, Italy) that is the regional referral centre for the diagnosis of Mycobacterium. We analysed retrospectively all consecutive extrapulmonary samples received between January 1, 2018 and October 31, 2020 from patients with high suspect of EPTB. Patient information, including age, sex, nationality, type of sample and microbiology results of routine TB diagnostic tests were collected from the laboratory information system. We presumed that the suspect of TB was generally high for tissues, lymph nodes and most of biological fluids samples due to the difficult of sample collection. The selection of pleural fluid samples that do not require an invasive sample collection was done in basis of medium/high clinical suspect indicated in the request forms received in the laboratory. Any case with positive culture for $M$. tuberculosis was considered confirmed EPTB whereas any sample with negative culture for $M$. tuberculosis after 42 days of incubation was confirmed negative. Samples collected from patients receiving antituberculosis therapy or previously diagnosed as having TB were excluded from the analysis. No data of HIV status was available.

\subsection{Sample Processing and TB Diagnostic Analysis}

The collected EPTB specimens included: biological fluids, pus and abscess, tissues, lymph nodes and aspirates from extrapulmonary sites. No less than $2 \mathrm{~mL}$ extrapulmonary fluids specimens were collected. Tissues were first homogenized by a tissue grinder and resuspended in $5 \mathrm{~mL}$ of sterile saline and then processed like other specimens. EPTB specimens with more than $5 \mathrm{~mL}$ were centrifuged at $3000 \mathrm{~g}$ for $15 \mathrm{~min}$ and the sediments were resuspended in $2 \mathrm{~mL}$ PBS buffer ( $\mathrm{pH}$ 6.8) (Becton Dickinson, BBL MycoPrep Kit) via vortexing for $30 \mathrm{~s}$. Specimens were pre-treated with $\mathrm{N}$-acetyl-L-cysteine- $\mathrm{NaOH}$ for $20 \mathrm{~min}$ at room temperature, and then neutralized with PBS buffer (50 mL final volume). Cerebrospinal fluid $(\mathrm{CSF})$ was centrifuged at $12,000 \times \mathrm{g}$ for $10 \mathrm{~min}$. The pellet was resuspended 
in PBS and treated without prior decontamination. After centrifugation at 3000 $\mathrm{g}$ for $20 \mathrm{~min}$, the remaining volume was processed as follows: 1) smear microscopy for acid-fast bacilli (AFB) with Ziehl-Neelsen (ZN) method, 2) Xpert ${ }^{\mathbb{B}}$ MTB/ RIF Ultra assay, 3) mycobacterial culture in solid (Löwenstein-Jensen) and liquid media (BACTEC MGIT 960; Becton Dickinson, Sparks, MD, USA). For liquid culture, the sediments were inoculated into a mycobacteria growth indicator tube (MGIT) to which an enrichment supplement (OADC) and an antibiotic mixture (PANTA) were added. The MGIT tubes were placed into the Bactec MGIT 960 system (BACTEC MGIT 960, Becton Dickinson, Sparks, MD, USA), which is a fluorescence-based detection instrument. Liquid and solid cultures were continuously monitored for bacterial growth for 42 days. The rapid identification of MTB isolated from solid or liquid cultures was performed by immune chromatographic assay BIO-LINE SD Ag MPT64 TB test (Standard Diagnostics, Yonginsi, Gyeonggi-do, Republic of Korea). Drug susceptibility test (DST) to first-line drugs was done using the Bactec MGIT 960 system.

\subsection{Microscopy}

To detect AFB, smears from clinical samples were stained by the $\mathrm{ZN}$ stain and examined under the oil immersion objective lens of the microscope $(\times 100)$.

\subsection{Xpert MTB/RIF Ultra Assay}

Sample reagent was added as per protocol's instructions in a 3:1 ratio and incubated for $15 \mathrm{~min}$ at room temperature. Two millilitres of the liquefied sample were transferred into the Xpert MTB/RIF Ultra cartridge and loaded into the Gene Xpert system. The semiquantitative scale for Xpert Ultra results was: trace, very low, low, medium, or high.

\subsection{Statistical Analysis}

Culture was used as the reference standard to calculate the sensitivity, specificity and accuracy of Xpert Ultra assay. A $p$ value of less than 0.05 was considered statistically significant. Confidence intervals for sensitivity, specificity and accuracy are "exact" Clopper-Pearson confidence intervals.

\subsection{Ethical Statement}

This study was retrospective without interaction with patients and all patients' information was de-identified prior to analysis. Therefore, institutional ethics committee approval was not required.

\section{Results}

A total of 845 non-respiratory specimens were received for routine diagnostics from January 2018 to October 2020. 239 pleural fluids were excluded due to a low clinical suspect of TB. Finally, a total of 606 samples (449 Italian natives, 157 foreign-born people) were analysed for this study: 456 biological fluids (75.2\%), 
57 tissues (9.4\%), 53 pus and abscess (8.7\%) and 40 lymph nodes and aspirates (6.6\%) (Figure 1, Figure 2). All these samples have culture (solid and liquid), $\mathrm{ZN}$ microscopy and Ultra results. Among these samples, 45 were culture positive, whereas 561 remained negative. Of 45 culture-positive, only 7 were smear positive and 38 were smear negative. Of the 561 culture-negative, $\mathrm{ZN}$ microscopy detected negative results in all these cases. The overall sensitivity of smear examination was only $15.6 \%$ (95\% CI 6.5 - 29.5) and the overall specificity was 100\% (95\% CI 99.3 - 100) (Figure 3). The positive predictive value (PPV) and the negative predictive value (NPV) of $\mathrm{ZN}$ microscopy was $100 \%$ and $93.7 \%$ (95\% CI 92.9 - 94.4) respectively. The accuracy of ZN microscopy was $93.7 \%$ (95\% CI 91.5 - 95.5). Of 45 culture-positive, Ultra detected positive results in 43 cases and 2 cases resulted negatives. Among the 561 culture-negative, Ultra detected negative results in 547 cases, 14 cases were detected positives. The positive results (true positive and false positive) of Ultra were categorised as follows: high $(n=3)$, medium $(n=9)$, low $(n=16)$, very low $(n=20)$ and trace $(n=9)$. Among the positive results of Ultra assay, only two samples detected rifampicin resistance (RIF-R), and both were in concordance with the DST results. The overall sensitivity and specificity of Ultra were 95.6\% (95\% CI 84.8 - 99.5) and 97.5\% (95\% CI 95.8 - 98.6) respectively. The positive predictive value (PPV) and the negative predictive value (NPV) of Ultra assay was 75.4\% (95\% CI 64.6 83.8 ) and $99.6 \%$ (95\% CI 98.6 - 99.9) respectively. The sensitivity and specificity of Ultra assay did not vary significantly among different clinical samples. With

\section{Specimens distribution}

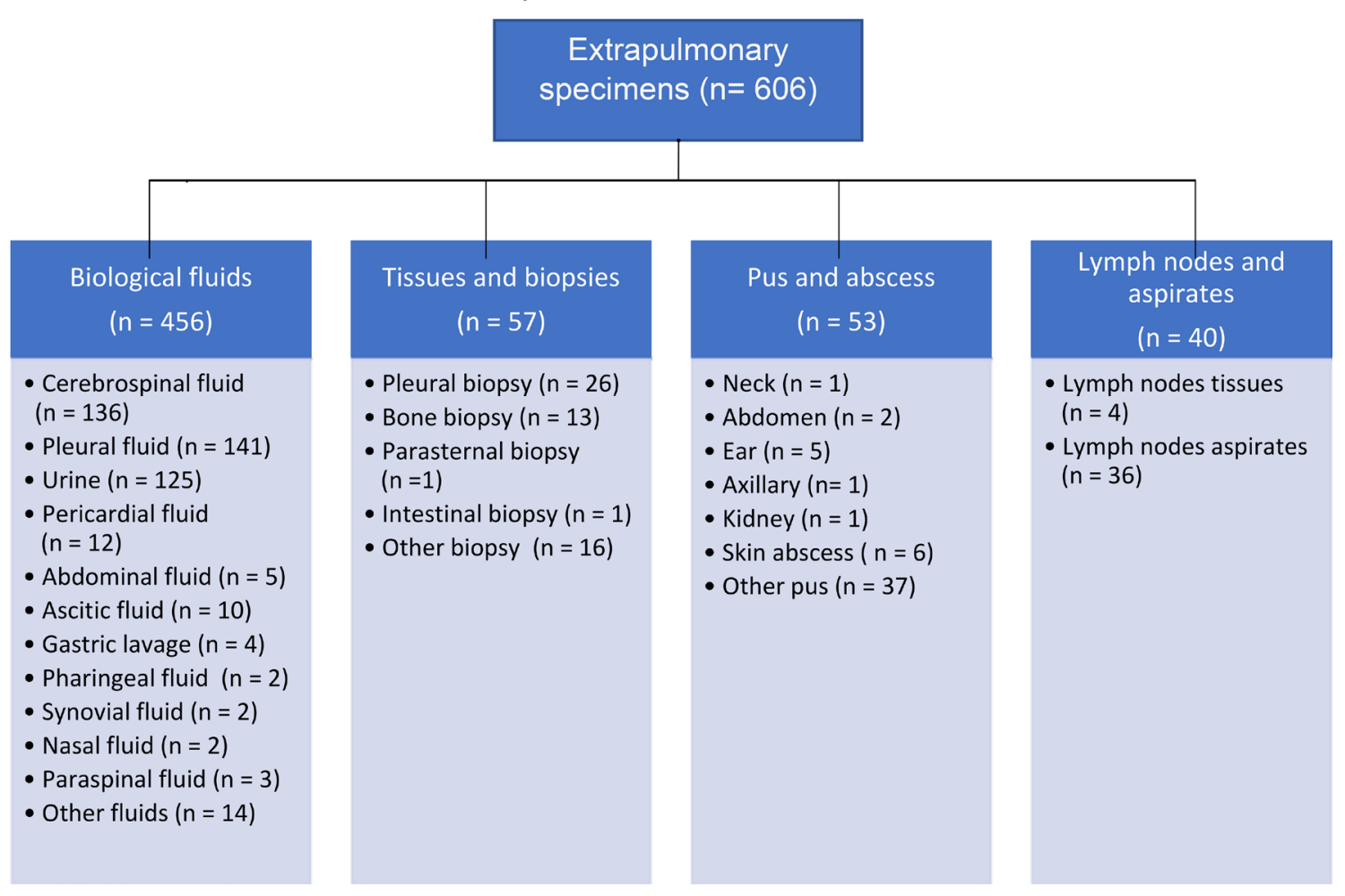

Figure 1. Distribution of extrapulmonary specimens included in the study. 


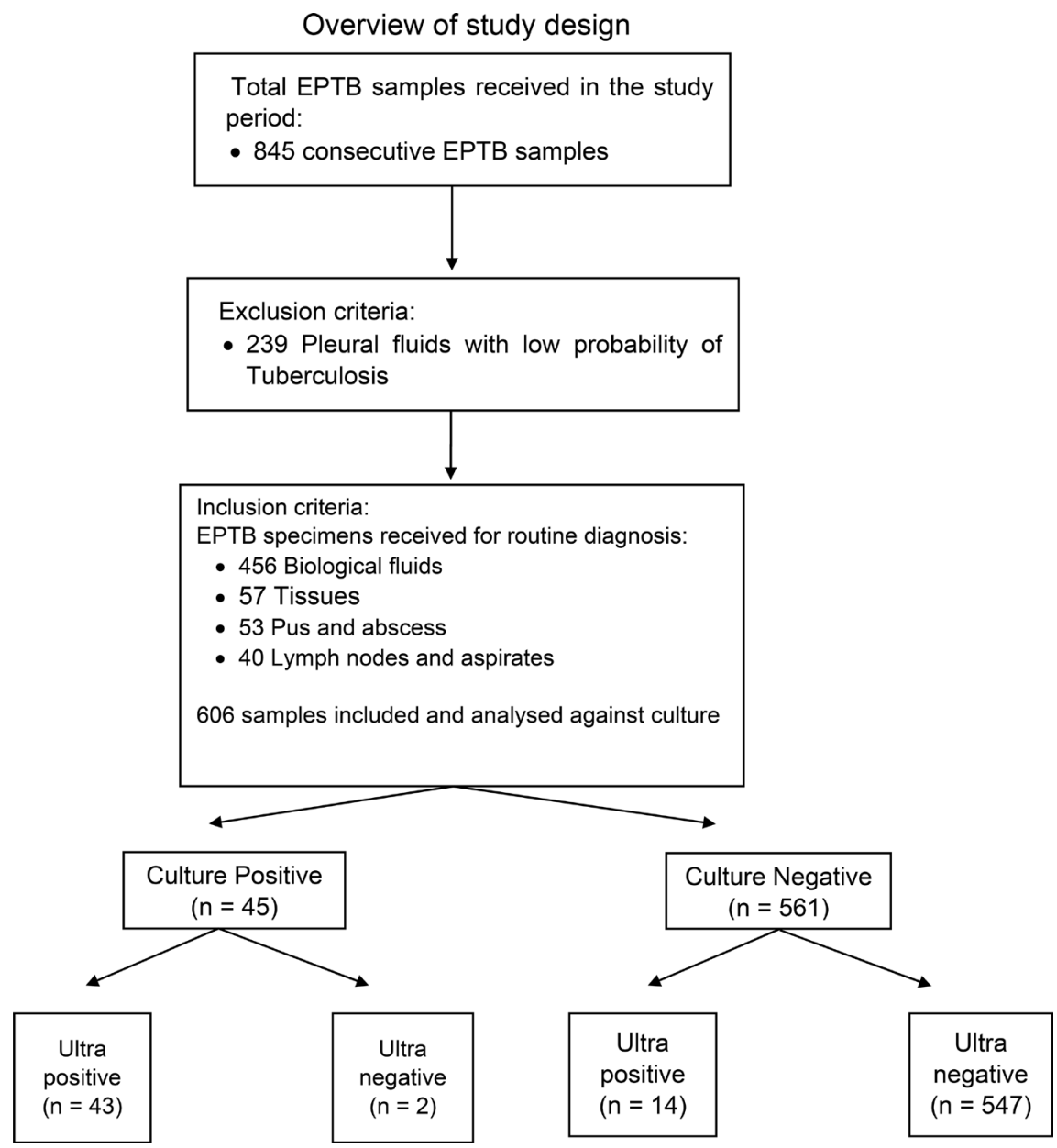

Figure 2. Study design and samples inclusion flowchart (EPTB extrapulmonary tuberculosis, Ultra GeneXpert MTB/Rif Ultra).

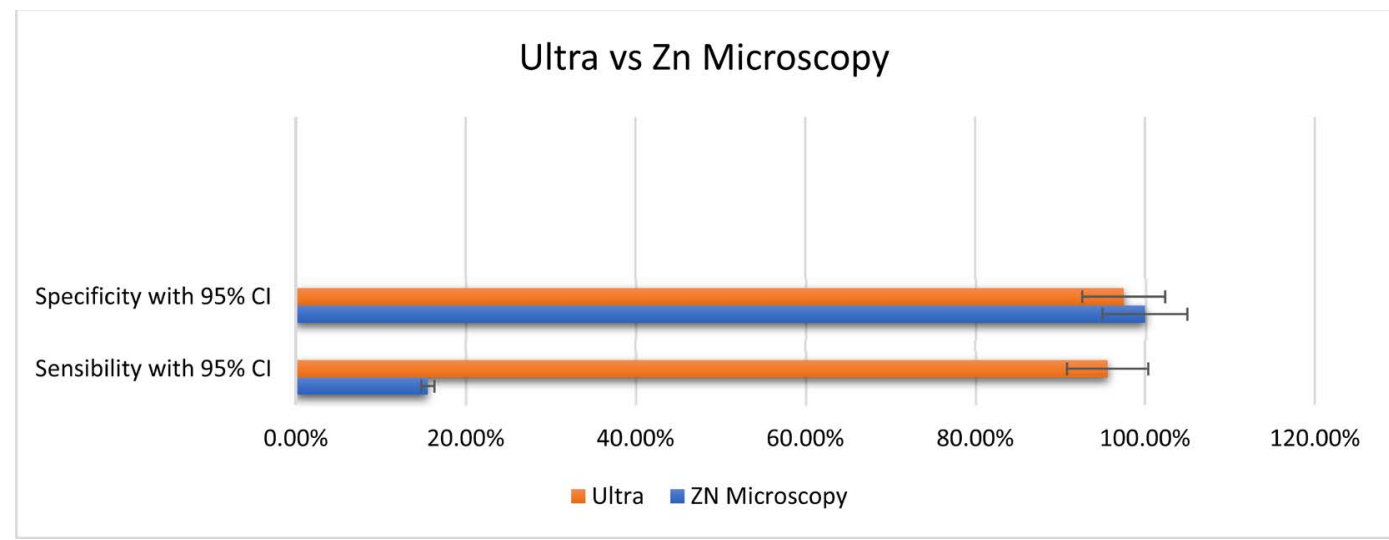

Figure 3. Sensitivity and specificity of Xpert MTB/Rif Ultra compared to Zn (Ziehl-Neelsen) smear Microscopy (CI, confidence intervals).

respect to the different specimen types, sensitivity of Ultra assay was $93.3 \%$ (95\% CI 68 - 99.8) for biological fluids, 90\% (95\% CI 55.5 - 99.7) for tissues, $100 \%$ (95\% CI 69.1 - 100) for lymph nodes, $100 \%$ (95\% CI 69.1 - 100) for pus and abscess. Specificity of Ultra assay was $98.2 \%$ (95\% CI 96.5 - 99.2) for biological flu- 
ids, 95.7\% (95\% CI 85.5 - 99.5) for tissues, 93.3\% (95\% CI 77.9 - 99.1) for lymph nodes, 95.3\% (95\% CI 84.2 - 99.4) for pus and abscess. The study has shown an accuracy of Gene Xpert Ultra 97.4\% (95\% CI 95.7 - 98.5) (Figure 4, Table 1).

\section{Discussion}

The study focuses on the evaluation of the diagnostic performance of Ultra assay using data of suspected EPTB samples under clinical routine conditions in a low-prevalence setting. So far, only few studies have investigated the use of Ultra in a non-endemic setting [17] [18] [19]. Two of these studies have used defrosted samples and the conditions of cryopreservation could have influenced any variations of sensitivity [17] [18], instead, for our study we have used a retrospective analysis method based on criteria of sample inclusion that reflects the true population-based investigation in a low prevalence setting.

In the present study, Ultra showed an overall sensitivity of $95.6 \%$ on extrapulmonary samples when culture is used as reference standard. The observed sensitivity

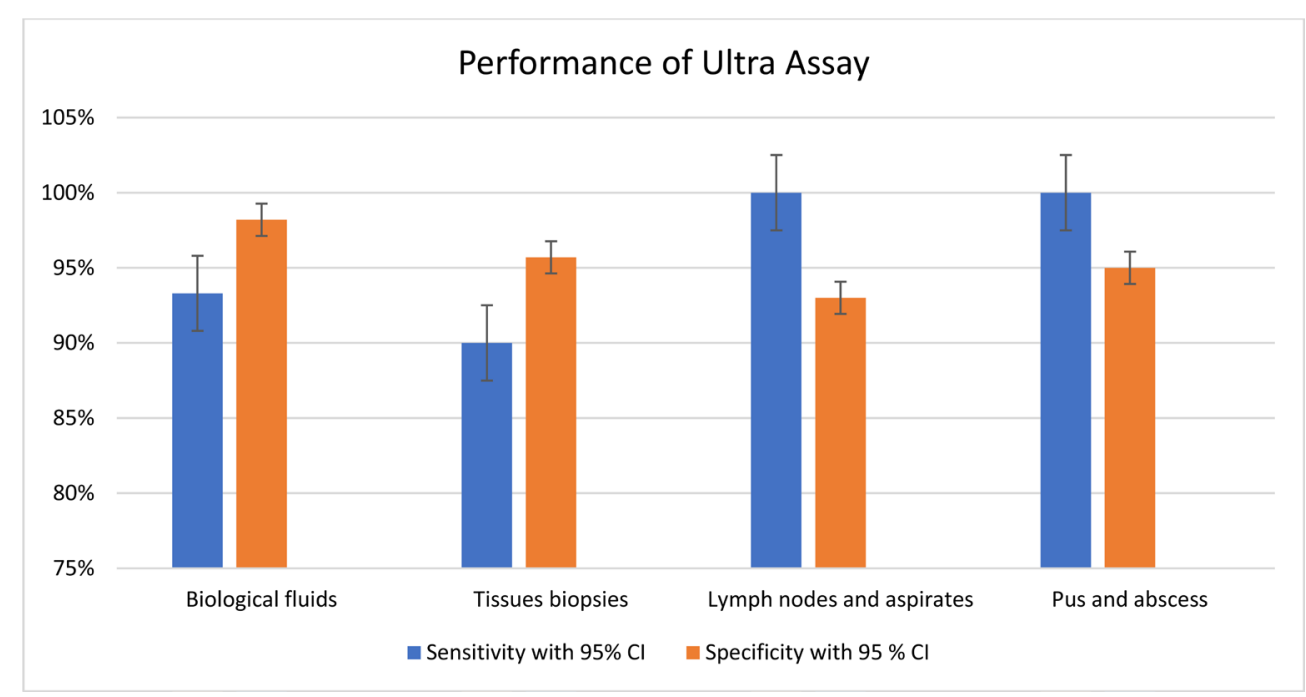

Figure 4. Sensitivity and specificity of Xpert MTB/Rif Ultra for different specimens; biological fluids, tissues and biopsies, lymph nodes and aspirates, pus and abscess (CI, confidence intervals).

Table 1. Performance of Xpert MTB/RIF Ultra using culture as reference standard. 95\% confidence intervals for sensitivity, specificity and accuracy were calculated using Clopper-Pearson intervals. (CI confidence interval, PPV positive predictive value, NPV negative predictive value).

\begin{tabular}{|c|c|c|c|c|c|c|c|c|c|}
\hline \multirow{3}{*}{ Specimen material } & \multicolumn{4}{|c|}{$\mathrm{N}^{\bullet}$ of specimens } & \multirow{2}{*}{\multicolumn{5}{|c|}{ Performance (\%) }} \\
\hline & \multicolumn{2}{|c|}{ EPTB culture positive } & \multicolumn{2}{|c|}{ EPTB culture negative } & & & & & \\
\hline & $\begin{array}{c}\text { Ultra } \\
\text { positive }\end{array}$ & $\begin{array}{c}\text { Ultra } \\
\text { negative }\end{array}$ & $\begin{array}{c}\text { Ultra } \\
\text { positive }\end{array}$ & $\begin{array}{c}\text { Ultra } \\
\text { negative }\end{array}$ & $\begin{array}{l}\text { Sensitivity } \\
(95 \% \text { CI) }\end{array}$ & $\begin{array}{l}\text { Specificity } \\
(95 \% \text { CI })\end{array}$ & $\begin{array}{c}\text { PPV } \\
(95 \% \mathrm{CI})\end{array}$ & $\begin{array}{c}\text { PPN } \\
(95 \% \mathrm{CI})\end{array}$ & $\begin{array}{l}\text { Accuracy } \\
(95 \% \mathrm{CI})\end{array}$ \\
\hline Biological fluids $(\mathrm{n}=456)$ & 14 & 1 & 8 & 433 & $93.3 \%$ & $98.2 \%$ & $63.6 \%$ & $99.8 \%$ & $98.0 \%$ \\
\hline Tissues biopsies $(\mathrm{n}=57)$ & 9 & 1 & 2 & 45 & $90.0 \%$ & $95.7 \%$ & $81.8 \%$ & $97.8 \%$ & $94.7 \%$ \\
\hline Lymph nodes and aspirates $(n=40)$ & 10 & 0 & 2 & 28 & $100 \%$ & $93.3 \%$ & $83.3 \%$ & $100 \%$ & $95.0 \%$ \\
\hline Pus and abscess $(n=53)$ & 10 & 0 & 2 & 41 & $100 \%$ & $95.3 \%$ & $83.3 \%$ & $100 \%$ & $96.2 \%$ \\
\hline Total $(\mathrm{n}=606)$ & 43 & 2 & 14 & 547 & $95.6 \%$ & $97.5 \%$ & $75.4 \%$ & $99.6 \%$ & $97.4 \%$ \\
\hline
\end{tabular}


of Xpert ${ }^{\circledR}$ MTB/RIF Ultra assay is in line with other published studies in which reported sensitivities ranged from $69.2 \%$ to $100 \%$ [17] [19] [20].

The highest sensitivity was obtained in samples of pus and abscess (100\%), lymph nodes and aspirates (100\%), following by biological fluids (93.3\%) and tissues $(90 \%)$. The results for tissues are in line with precedent studies where the sensitivities reported were between $87 \%$ - 95\% [4] [10]. We found a higher sensitivity for lymph nodes and pus specimens in respect to other studies where the sensitivity reported was generally between 90\% - 94\% [10] [21] and 65\% - 95\% [10] [16] respectively. Our results showed a general increase of sensitivity for all types of extrapulmonary specimens, contributing to improvement in the diagnosis of Tuberculosis in paucibacillary samples. These results reflect the increasing sensibility of Ultra assay due to the incorporation of multi-copy IS1081 and IS6110 insertion enable to detect lowest bacillary load of MTB [9].

In our contest, Ultra significantly improved MTB detection in EPTB samples compared to ZN microscopy that showed a sensibility of only $15.6 \%$. Indeed, of 45 EPTB culture-positive, only 7 were detected by ZN microscopy while 43 were detected by Ultra assay. The high sensitivity of Ultra can facilitate the diagnosis of extrapulmonary forms of Tuberculosis featured by an almost complete negativity of smear microscopy [19] and in only two hours compared to 42 days of culture.

The specificity of Ultra in our study is high (97.5\%). This result is in concordance with the specificities (97\% - 100\%) found in other low TB prevalent settings where culture was used as reference standard [10] [19]. In low TB prevalent settings, even a small reduction in specificity could increase the rate of false positive cases. For this reason, Ultra should be performed on pre-selected samples with a high clinical suspect of TB disease [18]. In other studies, it has been reported that the increase in sensitivity with Ultra respect Xpert, specifically in patients with a history of TB [12] [22] and patients with meningitis [23] came at the expense of a decrease in specificity. Although we excluded samples collected from patients receiving anti-tuberculosis therapy or previously diagnosed as having TB, we found 14 false positive results with Ultra assay. The 14 false-positive results were categorised as low $(\mathrm{n}=3)$, very low $(\mathrm{n}=8)$ and trace $(\mathrm{n}=3)$. No medium or high results were found. Due to the difficulty in obtaining qualified pathological specimens, not repeated tests were done on tissues, lymph nodes and pus samples. On the 14 false positive, 7 were urine samples and were repeated. Out of 7 repeat tests, 6 of them turned out as not detected. Only one urine sample confirmed the positivity of Ultra assay and it was clinically diagnosed as pyelonephritis, an inflammation of the kidney, typically due to a bacterial infection. These results, although too small, suggest a reclassification of these samples as confirmed tuberculosis negative.

The incidence of MDR-TB in our country, is low [1] [2]. Indeed, only two samples presented mutations related to RIF-R with Ultra assay, both confirmed with DST. One of them was a liquor. In this case, Ultra assay was essential to the 
rapid diagnosis of Tuberculous meningitis and the initial treatment of TB with $2^{\text {nd }}$ line anti-tuberculosis drugs. One of the strengths of Ultra assay is to simultaneously detect MTC and RIF-R is but the inconsistency of our data does not permit to evaluate the overall performance of Ultra assay on MDR-TB EPTB samples.

This study has some limitations. We conducted a retrospective investigation based on a short observation period and with a limited number of sample sizes that can lead to uncertain the sensitivity and specificity estimates. However, we used data over a period of three years, considering the new cartridge was installed in our setting at the end of 2017. Further studies, including clinical data, are needed to better investigate the accuracy of Ultra assay.

\section{Conclusion}

To conclude, our results confirm Ultra assay to have a good performance in the diagnosis of EPTB in our setting. More studies are required to investigate the decrease of specificity of Ultra assay on the different types of extrapulmonary samples and the incidence and clinical consequence of false-positives results in countries with low TB incidence.

\section{Conflicts of Interest}

The authors declare no conflicts of interest regarding the publication of this paper.

\section{References}

[1] World Health Organization (2020) Global Tuberculosis Report, 2020. WHO, Geneva.

[2] WHO Regional Office for Europe/European Centre for Disease Prevention and Control (2019) Tuberculosis Surveillance and Monitoring in Europe 2019-2017 Data. WHO Regional Office for Europe, Copenhagen.

[3] Lee, J.Y. (2015) Diagnosis and Treatment of Extrapulmonary Tuberculosis. Tuberculosis and Respiratory Diseases, 78, 47. https://doi.org/10.4046/trd.2015.78.2.47

[4] Wu, X., Tan, G., Gao, R., Yao, L., Bi, D., Guo, Y., et al. (2019) Assessment of the Xpert MTB/RIF Ultra Assay on Rapid Diagnosis of Extrapulmonary Tuberculosis. International Journal of Infectious Diseases, 81, 91-96. https://doi.org/10.1016/j.ijid.2019.01.050

[5] Suzana, S., Ninan, M.M., Gowri, M., Venkatesh, K., Rupali, P. and Michael, J.S. (2016) Xpert MTB/Rif for the Diagnosis of Extrapulmonary Tuberculosis-An Experience from a Tertiary Care Centre in South India. Tropical Medicine \& International Health, 21, 385-392. https://doi.org/10.1111/tmi.12655

[6] Lawn, S.D. and Nicol, M.P. (2011) Xpert ${ }^{\circledR}$ MTB/RIF Assay: Development, Evaluation and Implementation of a New Rapid Molecular Diagnostic for Tuberculosis and Rifampicin Resistance. Future Microbiology, 6, 1067-1082. https://doi.org/10.2217/fmb.11.84

[7] Kohli, M., Schiller, I., Dendukuri, N., Dheda, K., Denkinger, C.M., Schumacher, S.G., et al. (2018) Xpert ${ }^{\circledR}$ MTB/RIF Assay for Extrapulmonary Tuberculosis and Ri- 
fampicin Resistance. Cochrane Database of Systematic Reviews, No. 8, CD012768. https://doi.org/10.1002/14651858.CD012768.pub2

[8] WHO (2017) WHO Meeting Report of a Technical Expert Consultation: Non-Inferiority Analysis of Xpert MTB/RIF Ultra Compared to Xpert MTB/RIF. World Health Organization, Geneva.

[9] Chakravorty, S., Simmons, A.M., Rowneki, M., Parmar, H., Cao, Y., Ryan, J., et al. (2017) The New Xpert MTB/RIF Ultra: Improving Detection of Mycobacterium tuberculosis and Resistance to Rifampin in an Assay Suitable for Point-of-Care Testing. MBio, 8, e00812-17. https://doi.org/10.1128/mBio.00812-17

[10] Perez-Risco, D., Rodriguez-Temporal, D., Valledor-Sanchez, I. and Alcaide, F. (2018) Evaluation of the Xpert MTB/RIF Ultra Assay for Direct Detection of Mycobacterium tuberculosis Complex in Smear-Negative Extrapulmonary Samples. Journal of Clinical Microbiology, 56, e00659-18.

https://doi.org/10.1128/JCM.00659-18

[11] World Health Organization (2017) WHO Meeting Report of a Technical Expert Consultation: Non-Inferiority Analysis of Xpert MTB/RIF Ultra Compared to Xpert MTB/RIF.

https://apps.who.int/iris/bitstream/handle/10665/254792/WHO-HTM-2017.04-eng. pdf; sequence $=1$

[12] Dorman, S.E., Schumacher, S.G., Alland, D., Nabeta, P., Armstrong, D.T., King, B., et al. (2018) Xpert MTB/RIF Ultra for Detection of Mycobacterium tuberculosis and Rifampicin Resistance: A Prospective Multicentre Diagnostic Accuracy Study. The Lancet Infectious Diseases, 18, 76-84. https://doi.org/10.1016/S1473-3099(17)30691-6

[13] Wang, G., Wang, S., Jiang, G., Yang, X., Huo, F., Ma, Y., et al. (2019) Xpert MTB/ RIF Ultra Improved the Diagnosis of Paucibacillary Tuberculosis: A Prospective Cohort Study. Journal of Infection, 78, 311-316. https://doi.org/10.1016/j.jinf.2019.02.010

[14] Kolia-Diafouka, P., Carrère-Kremer, S., Lounnas, M., Bourdin, A., Kremer, L., Van de Perre, P., et al. (2019) Detection of Mycobacterium tuberculosis in Paucibacillary Sputum: Performances of the Xpert MTB/RIF Ultra Compared to the Xpert MTB/ RIF, and IS6110 PCR. Diagnostic Microbiology and Infectious Disease, 94, 365-370. https://doi.org/10.1016/j.diagmicrobio.2019.02.008

[15] Sekyere, J.O., Maphalala, N., Malinga, L.A., Mbelle, N.M. and Maningi, N.E. (2019) A Comparative Evaluation of the New Genexpert MTB/RIF Ultra and Other Rapid Diagnostic Assays for Detecting Tuberculosis in Pulmonary and Extra Pulmonary Specimens. Scientific Reports, 9, Article No. 16587.

https://doi.org/10.1038/s41598-019-53086-5

[16] Sun, Q., Wang, S., Dong, W., Jiang, G., Huo, F., Ma, Y., et al. (2019) Diagnostic Value of Xpert MTB/RIF Ultra for Osteoarticular Tuberculosis. Journal of Infection, 79, 153 158. https://doi.org/10.1016/j.jinf.2019.06.006

[17] Bisognin, F., Lombardi, G., Lombardo, D., Re, M.C. and Dal Monte, P. (2018) Improvement of Mycobacterium tuberculosis Detection by Xpert MTB/RIF Ultra: A Head-to-Head Comparison on Xpert-Negative Samples. PLoS ONE, 13, e0201934. https://doi.org/10.1371/journal.pone.0201934

[18] Hoel, I.M., Syre, H., Skarstein, I. and Mustafa, T. (2020) Xpert MTB/RIF Ultra for Rapid Diagnosis of Extrapulmonary Tuberculosis in a High-Income Low-Tuberculosis Prevalence Setting. Scientific Reports, 10, Article No. 13959.

https://doi.org/10.1038/s41598-020-70613-x 
[19] Piersimoni, C., Gherardi, G., Gracciotti, N. and Pocognoli, A. (2019) Comparative Evaluation of Xpert MTB/RIF and the New Xpert MTB/RIF Ultra with Respiratory and Extra-Pulmonary Specimens for Tuberculosis Case Detection in a Low Incidence Setting. Journal of Clinical Tuberculosis and Other Mycobacterial Diseases, 15, Article ID: 100094. https://doi.org/10.1016/j.jctube.2019.100094

[20] López-Roa, P., Martin-Higuera, C., Ruiz-Serrano, M.J., Toro, C., Tato, M., Simon, M., et al. (2021) Performance of Xpert MTB/RIF Ultra Assay on Respiratory and Extra-Respiratory Samples in a High-Resource Setting with a Low Tuberculosis Prevalence. Diagnostic Microbiology and Infectious Disease, 99, Article ID: 115235. https://doi.org/10.1016/j.diagmicrobio.2020.115235

[21] Antel, K., Oosthuizen, J., Malherbe, F., Louw, V.J., Nicol, M.P., Maartens, G., et al. (2020) Diagnostic Accuracy of the Xpert MTB/Rif Ultra for Tuberculosis Adenitis. BMC Infectious Diseases, 20, 33. https://doi.org/10.1186/s12879-019-4749-X

[22] Theron, G., Venter, R., Smith, L., Esmail, A., Randall, P., Sood, V., et al. (2018) FalsePositive Xpert MTB/RIF Results in Retested Patients with Previous Tuberculosis: Frequency, Profile, and Prospective Clinical Outcomes. Journal of Clinical Microbiology, 56, e01696-17. https://doi.org/10.1128/JCM.01696-17

[23] Bahr, N.C., Nuwagira, E., Evans, E.E., Cresswell, F.V., Bystrom, P.V., Byamukama, A., et al. (2018) Diagnostic Accuracy of Xpert MTB/RIF Ultra for Tuberculous Meningitis in HIV-Infected Adults: A Prospective Cohort Study. The Lancet Infectious Diseases, 18, 68-75. https://doi.org/10.1016/S1473-3099(17)30474-7 